\title{
Stable Controller Design for Linear Systems
}

\author{
Guang-Hong Yang Jian Liang Wang and Yeng Chai Soh \\ School of Electrical and Electronic Engineering \\ Nanyang Technological University, Nanyang Avenue, Singapore 639798 \\ Emails:egyang@ntu.edu.sg, ejlwang@ntu.edu.sg, eycsoh@ntu.edu.sg \\ James Lam \\ Department of Mechanical Engineering, University of Hong Kong \\ Pokfulam Road, Hong Kong, China. Email:jlam@hku.hk.
}

\begin{abstract}
This paper is concerned with the problem of suboptimal stable mixed $H_{2} / H_{\infty}$ control for linear timeinvariant systems. The designed controllers are required to satisfy a prescribed $H_{\infty}$ performance bound or a prescribed degree of stability. By reducing the stable controller synthesis problem to a multiobjective state feedback control problem for two different state models, sufficient conditions for the solvability of the considered problem are given in terms of solutions to algebraic Riccati equations and matrix inequalities. LMI-based iterative algorithms are developed to solve the stable controller synthesis problem. All of the proposed algorithms are shown to be convergent. An example is given to illustrate the proposed methods.
\end{abstract}

Keywords: Linear systems; dynamic output feedback; mixed $\mathrm{H}_{2} / \mathrm{H}_{\infty}$ control; stable controller; LMI.

\section{Introduction}

The problem of designing a stable controller to stabilize a given plant with some performance specifications has been extensively investigated by a number of authors, see [2], [5], [6]-[8] [11], [13]-[18]. In [16], it has been shown that a necessary and sufficient condition for the existence of a stable stabilizing controller is the parity interlacing property. A plant $P$ is said to satisfy the parity interlacing property if the number of poles of $P$ between any pair of real right half-plane blocking zeros is even. Some procedures for constructing stable stabilizing controllers are given in $[12,16]$, which involve the construction of a unit in $H_{\infty}$ satisfying certain interpolation conditions that may result in very large order controllers. For the stable $H_{\infty}$ controller design, a method using a state space approach is proposed in [11]. In [6], sufficient conditions are also obtained for the synthesis of SISO finite dimensional suboptimal stable $H_{\infty}$ controllers by converting the problem into a Nevanlinna-Pick interpolation problem. In [18], a suffi- cient condition for the existence of a stable suboptimal $H_{\infty}$ controller is derived in terms of positive definite stabilizing solution to a certain algebraic Riccati equation. However, the order of the designed controller is two times that of the plant. A result proposing stable $H_{\infty}$ controllers which have the same order as the plant is given in [17], and the $H_{\infty}$ performance of the stable $H_{\infty}$ controllers is investigated. More recently, a method of designing a stable $H_{\infty}$ controller is also given by using the Riccati equation approach, where the designed controller is of the same order as that of the plant, and satisfies the same $H_{\infty}$-norm bound as that of the resulting closed-loop system [2]. For the problem of designing stable $\mathrm{H}_{2}$ controller, [5] presents an algorithm which requires the minimization of a nonlinear objective function with nonlinear inequality constraints. Based on the cost function modification, a sufficient condition for the stable $\mathrm{H}_{2}$ control problem is given in [13]. The method is also extended to the design of a stable controller for mixed $H_{2} / H_{\infty}$ problem [8].

This paper will be concerned with the problem of designing suboptimal stable mixed $\mathrm{H}_{2} / \mathrm{H}_{\infty}$ controllers for linear time-invariant systems. The controller to be designed is required to satisfy a prescribed $H_{\infty}$ performance bound or a prescribed degree of stability. The stable controller synthesis problem is reduced to a multiobjective state feedback control problem for two different state models. New sufficient conditions for the solvability of the problem are given in terms of solutions to Riccati equations and matrix inequalities by solving the multiobjective state feedback control problem. Iterative algorithms are developed to solve the stable controller synthesis problem. All of the proposed algorithms are shown to be convergent, and the numerical example illustrates the advantage of the proposed algorithms. The paper is organized as follows. Section 2 formulates the problem under consideration and gives some preliminaries. The suboptimal stable mixed 
$H_{2} / H_{\infty}$ control problem is addressed in Section 3. A numerical example is given in Section 4.

\section{Problem statement and preliminaries} Consider a linear time-invariant system $\Sigma$ described by equations

$$
\begin{aligned}
\Sigma: \quad \dot{x} & =A x+B_{1} w+B_{2} u \\
z_{0} & =C_{0} x+D_{0} u \\
z_{1} & =C_{1} x+D_{1} u \\
y & =C_{2} x+D_{2} w
\end{aligned}
$$

where $x \in R^{n}$ is the state, $u \in R^{m}$ is the control input, $y \in R^{q}$ is the measured output, $w \in R^{r}$ is the disturbance inputs, and $z_{0} \in R^{r_{0}}$ and $z_{1} \in R^{r_{1}}$ are the outputs to be regulated. The dynamic output feedback controller $K$ is given by

$$
K: \quad \begin{aligned}
\dot{\xi} & =A_{K} \xi+B_{K} y \\
u & =C_{K} \xi+D_{K} y
\end{aligned}
$$

where $\xi \in R^{v}$. The resulting closed-loop system $\Sigma_{c}$ with the controller $K$ is described as follows

$$
\begin{aligned}
\Sigma_{c}: \quad \dot{x}_{e} & =A_{e} x_{e}+B_{e} w \\
z_{0} & =C_{0 e} x_{e}+J_{0 e} w \\
z_{1} & =C_{1 e} x_{e}+J_{1 e} w
\end{aligned}
$$

where $x_{e}=\left[\begin{array}{ll}x^{T} & \xi^{T}\end{array}\right]^{T}$,

$$
\begin{aligned}
A_{e} & =\left[\begin{array}{cc}
A+B_{2} D_{K} C_{2} & B_{2} C_{K} \\
B_{K} C_{2} & A_{K}
\end{array}\right] \\
B_{e} & =\left[\begin{array}{c}
B_{1}+B_{2} D_{K} D_{2} \\
B_{K} D_{2}
\end{array}\right] \\
C_{0 e} & =\left[\begin{array}{ll}
C_{0}+D_{0} D_{K} C_{2} & D_{0} C_{K}
\end{array}\right], \quad J_{0 e}=D_{0} D_{K} D_{2} \\
C_{1 e} & =\left[\begin{array}{lll}
C_{1}+D_{1} D_{K} C_{2} & D_{1} C_{K}
\end{array}\right], \quad J_{1 e}=D_{1} D_{K} D_{2}
\end{aligned}
$$

By [9], the mixed $H_{2} / H_{\infty}$ performance measure $J(\Sigma, K, \gamma)$ of the stable system $\Sigma_{c}$ is defined as follows

$$
J(\Sigma, K, \gamma)= \begin{cases}\infty, & \text { if } J_{0 e} \neq 0 \\ \operatorname{Trace}\left(C_{0 e} Y C_{0 e}^{T}\right), & \text { otherwise }\end{cases}
$$

where $Y \geq 0$ is the stabilizing solution to the following algebraic Riccati equation

$$
\begin{gathered}
A_{e} Y+Y A_{e}^{T}+\left(Y C_{1 e}^{T}+B_{e} J_{1 e}^{T}\right)\left(\gamma^{2}-J_{1 e} J_{1 e}^{T}\right)^{-1} \\
\times\left(Y C_{1 e}^{T}+B_{e} J_{1 e}^{T}\right)^{T}+B_{e} B_{e}^{T}=0
\end{gathered}
$$

Definition 2.1: A symmetric matrix $X_{0}$ is said to be a stabilizing solution to the Riccati equation $A^{T} X+$ $X A-X M X+N=0$ if it satisfies the Riccati equation and the matrix $A-M X_{0}$ is stable.

Then the problem under consideration is as follows.

Suboptimal $\beta$-stable mixed $H_{2} / H_{\infty}$ control problem: Given constants $\gamma>0, \alpha>0$ and $\beta>0$, find a stable controller $K$ with $\|K(s)\|_{\infty}<\beta$ such that the closed-loop system $\Sigma_{c}$ is internally stable and the mixed $H_{2} / H_{\infty}$ performance measure $J(\Sigma, K, \gamma)$ of the closed-loop system $\Sigma_{c}$ satisfies $J(\Sigma, K, \gamma)<\alpha$.

By imposing the constraint of an $H_{\infty}$ performance bound on the designed controller, the robust stability of the closed-loop system may be guaranteed. The problem of designing a stable $H_{\infty}$ controller to satisfy an $H_{\infty}$ performance was considered in [17], and [2] for the special case $\gamma=\beta$, respectively. When $\beta$ is sufficiently large, the above suboptimal $\beta$-stable mixed $H_{2} / H_{\infty}$ control problem becomes one of designing a stable $H_{2} / H_{\infty}$ controller. In the sequel, the more general problem of designing an $\mathrm{H}_{2} / \mathrm{H}_{\infty}$ controller with the prescribed degree of stability $r$ will also be addressed. If there is no constraints of $K$ being stable and $\|K\|_{\infty}<\beta$, then the problem is reduced to the mixed $H_{2} / H_{\infty}$ control problem considered in [9]. In particular, [9] has shown that for a given $\gamma>0$, the computation of the optimal mixed $\mathrm{H}_{2} / \mathrm{H}_{\infty}$ performance $J_{o p t}(\Sigma, \gamma)$ and the construction of a suboptimal compensator can be approached via convex optimization, where

$$
\begin{gathered}
J_{o p t}(\Sigma, \gamma)=\inf _{K}\left\{J(\Sigma, K, \gamma):\left\|T_{z_{1} w}(K)\right\|_{\infty}<\gamma\right. \text { and } \\
\left.A_{e} \text { is stable }\right\}
\end{gathered}
$$

with the transfer function $T_{z_{1} w}(K)$ being defined by

$$
T_{z_{1} w}(K)=J_{1 e}+C_{1 e}\left(s I-A_{e}\right)^{-1} B_{e}
$$

The following assumptions will be used in the sequel. Assumption A1: The triple $\left(C_{2}, A, B_{2}\right)$ is stabilizable and detectable.

Assumption A2: The pair $\left(A, B_{1}\right)$ is stabilizable. Assumption A3: $D_{2}\left[\begin{array}{ll}B_{1}^{T} & D_{2}^{T}\end{array}\right]=\left[\begin{array}{ll}0 & I\end{array}\right]$.

3 Stable mixed $H_{2} / H_{\infty}$ controller design In this section, we will present new sufficient conditions for the solvability of the suboptimal stable mixed $H_{2} / H_{\infty}$ control problem, and an algorithm will be proposed to minimize the mixed $H_{2} / H_{\infty}$ performance $J(\Sigma, K, \gamma)$ under the constraints of $K$ being stable and $\|K\|_{\infty}<\beta$. The proofs are omitted, see [15] for the details.

Suppose that the Riccati equation

$$
A Y+Y A^{T}+Y\left(\frac{1}{\gamma^{2}} C_{1}^{T} C_{1}-C_{2}^{T} C_{2}\right) Y+B_{1} B_{1}^{T}=0
$$

has a stabilizing solution $Y \geq 0$ for $\gamma>0$. Define the auxiliary systems $\Sigma_{Y}$ and $\Sigma_{K Y}$ as follows:

$$
\begin{aligned}
& \Sigma_{Y}: \quad \quad \dot{x}=A_{11} x+B_{11} w+B_{22} u \\
& z_{Y 0}=C_{0} x+D_{0} u
\end{aligned}
$$




$$
\begin{aligned}
z_{Y 1} & =C_{1} x+D_{1} u \\
\Sigma_{K Y}: \quad \dot{x} & =A_{12} x+B_{11} w+B_{22} u \\
z_{K 1} & =u
\end{aligned}
$$

where $C_{0}, D_{0}, C_{1}$ and $D_{1}$ are the same as in (2) and $(3)$, and

$$
\begin{aligned}
& A_{11}=A+\frac{1}{\gamma^{2}} Y C_{1}^{T} C_{1} \\
& B_{11}=Y C_{2}^{T} \\
& B_{22}=B_{2}+\frac{1}{\gamma^{2}} Y C_{1}^{T} D_{1} \\
& A_{12}=A+\frac{1}{\gamma^{2}} Y C_{1}^{T} C_{1}-Y C_{2}^{T} C_{2}
\end{aligned}
$$

Then we have the following lemma.

Lemma 3.1: If there exists a common state feedback gain $C_{K}$ for the systems $\Sigma_{Y}$ and $\Sigma_{K Y}$ such that both $A_{11}+B_{22} C_{K}$ and $A_{12}+B_{22} C_{K}$ are stable, $J\left(\Sigma_{Y}, C_{K}, \gamma\right)<\alpha-\operatorname{Trace}\left(C_{0} Y C_{0}^{T}\right)$, and $\left\|T_{K Y}\left(s, C_{K}\right)\right\|_{\infty}<\beta$, then the dynamic output feedback controller $K$ described by (5) and (6) with the $C_{K}$ and

$$
A_{K}=A_{12}+B_{22} C_{K}, \quad B_{K}=B_{11}=Y C_{2}^{T}, \quad D_{K}=0
$$

solves the suboptimal $\beta$-stable mixed $H_{2} / H_{\infty}$ control problem, where

$$
T_{K Y}\left(s, C_{K}\right)=C_{K}\left(s I-A_{K}\right)^{-1} B_{K}
$$

The key idea here lies in the construction of the two auxiliary systems $\Sigma_{Y}$ and $\Sigma_{K Y}$, especially $\Sigma_{K Y}$. The system $\Sigma_{Y}$ is constructed in [9] for solving the suboptimal mixed $H_{2} / H_{\infty}$ output feedback control problem, which reduces the original outut feedback control problem into a state feedback control problem for $\Sigma_{Y}$. Notice that the designed controller can be given by (24), the matrices $A_{12}, B_{22}$ and $B_{11}$ are defined by (21)-(23) and only one parameter matrix $C_{K}$ is to be determined such that $\|K(s)\|_{\infty}<\beta\left(K(s)=T_{K Y}\left(s, C_{K}\right)\right)$. So the auxiliary system $\Sigma_{K Y}$ is constructed to reduce the problem of finding a gain $C_{K}$ such that $\|K(s)\|_{\infty}<\beta$ into a state feedback control problem for $\Sigma_{K Y}$. Thus, the suboptimal $\beta$-stable mixed $H_{2} / H_{\infty}$ control problem cab be reduced to the problem of finding a state feedback gain $C_{K}$ such that both the mixed $H_{2} / H_{\infty}$ state feedback control problem for $\Sigma_{Y}$ and the $H_{\infty}$ state feedback control problem for $\Sigma_{K Y}$ are solved by the same $C_{K}$. This implies that the stable controller synthesis problem can be reduced to the multiobjective state feedback control problem for the two different systems $\Sigma_{Y}$ and $\Sigma_{K Y}$. This approach differs from all previous existing approaches to the stable control design problem in [11], [6], [18], [17], [2], [13] and [8]

First, a sufficient condition for the solvability of the suboptimal $\beta$-stable mixed $H_{2} / H_{\infty}$ control problem is given in the following theorem.

Theorem 3.2: Consider the system $\Sigma$ described by equations (1)-(4). Let $\gamma>0, \alpha>0$ and $\beta>0$ be given constants. Suppose the following

(i) Assumptions A1-A3 hold;

(ii) The Riccati equation (14) has a stabilizing solution $Y \geq 0$;

(iii) There exist matrices $X>0, Q>0$ and $W$ such that the following inequalities hold

$$
\begin{gathered}
{\left[\begin{array}{cc}
M_{a} & X C_{1}^{T}+W^{T} D_{1}^{T} \\
C_{1} X+D_{1} W & -\gamma^{2} I
\end{array}\right]<0} \\
{\left[\begin{array}{cc}
-Q & C_{0} X+D_{0} W \\
X C_{0}^{T}+W^{T} D_{0}^{T} & -X
\end{array}\right]<0} \\
\operatorname{Trace}(Q)<\alpha-\operatorname{Trace}\left(C_{0} Y C_{0}^{T}\right) \\
{\left[\begin{array}{cc}
M_{b} & W^{T} \\
W & -\beta^{2} I
\end{array}\right]<0}
\end{gathered}
$$

where $A_{11}, B_{11}, B_{22}$ and $A_{12}$ are as defined in (20)-(23), $M_{a}=A_{11} X+X A_{11}^{T}+B_{22} W+W^{T} B_{22}^{T}+B_{11} B_{11}^{T}$ and $M_{b}=A_{12} X+X A_{12}^{T}+B_{22} W+W^{T} B_{22}^{T}+B_{11} B_{11}^{T}$. Then the dynamic output feedback controller $K$ described by (5) and (6) with (24), and

$$
C_{K}=W X^{-1}
$$

solves the suboptimal $\beta$-stable mixed $H_{2} / H_{\infty}$ control problem.

Note that (26)-(28) are the conditions for solving the suboptimal mixed $H_{2} / H_{\infty}$ control problem, and (29) for $\left\|T_{K Y}\left(s, C_{K}\right)\right\|_{\infty}<\beta$ (hence $\left.\|K(s)\|_{\infty}<\beta\right)$. For the case of designing a stable $\mathrm{H}_{2} / \mathrm{H}_{\infty}$ controller with the prescribed degree of stability $r$ (but without $\left\|T_{K Y}\left(s, C_{K}\right)\right\|_{\infty}<\beta$, i.e., $\left.\|K(s)\|_{\infty}<\beta\right)$, we have the following

Corollary 3.3: With all assumptions as in Theorem 3.2 except for (29) being replaced by

$$
\left(A_{12}+r I\right) X+X\left(A_{12}+r I\right)^{T}+B_{22} W+W^{T} B_{22}^{T}<0
$$

where $r \geq 0$ is a constant. Then the controller $K$ given by (5) and (6) with (30) and (24) is with the prescribed degree of stability $r$, and stabilizes the system $\Sigma$ with $J(\Sigma, K, \gamma)<\alpha$.

Remark 3.4: Theorem 3.2 presents a sufficient condition for the suboptimal $\beta$-stable mixed $H_{2} / H_{\infty}$ control problem in terms of solutions to Riccati equation (14) and matrix inequalities (26)-(29). For the fixed $Y \geq 0$ satisfying (14), (26)-(29) are LMIs, which can be solved by using the LMI Toolbox [4]. When there is no constraints of $K(s)$ being stable and $\|K(s)\|_{\infty}<\beta$ (i.e., remove (29)), Theorem 3.2 is reduced to the necessary and sufficient condition for the suboptimal mixed 
$H_{2} / H_{\infty}$ control problem in [9]. Corollary 3.3 presents a sufficient condition under which the designed mixed $H_{2} / H_{\infty}$ controller is with a prescribed degree of stability $r$.

It should be noted that the conditions (26)-(29) are given based on the assumption of existence of a common Lyapunov function for the two closed-loop systems from $\Sigma_{Y}$ and $\Sigma_{K X}$ with $u=C_{K} x$, which may result in some conservativeness. In the following, we will present another sufficient condition without this conservativeness. Denote

$$
\begin{gathered}
\Delta_{Y}\left(P_{1}, P_{10}, C_{K}, \gamma\right)=\left[\begin{array}{c}
\Delta_{Y 11}\left(P_{1}, P_{10}\right) \\
B_{11}^{T} P_{1} \\
C_{K}+R_{1}^{-1}\left(B_{22}^{T} P_{1}+D_{1}^{T} C_{1}\right)
\end{array}\right. \\
\left.\begin{array}{cc}
P_{1} B_{11} & C_{K}^{T}+\left(P_{1} B_{22}+C_{1}^{T} D_{1}\right) R_{1}^{-1} \\
0 & \gamma^{2} I \\
0 & -R_{1}^{-1}
\end{array}\right] \\
\Delta_{K Y}\left(P_{2}, P_{20}, C_{K}, \beta\right)=\left[\begin{array}{cc}
\Delta_{K Y 11}\left(P_{2}, P_{20}\right) & P_{2} B_{11} \\
B_{11}^{T} P_{2} & -\beta^{2} I \\
C_{K}+B_{22}^{T} P_{2} & 0 \\
C_{K}^{T}+P_{2} B_{22} \\
0 \\
-I
\end{array}\right]
\end{gathered}
$$

where

$$
\begin{aligned}
R_{1}= & D_{1}^{T} D_{1}>0 \\
\Delta_{Y 11}\left(P_{1}, P_{10}\right)= & P_{1}\left(A_{11}-B_{22} R_{1}^{-1} D_{1}^{T} C_{1}\right) \\
& +\left(A_{11}-B_{22} R_{1}^{-1} D_{1}^{T} C_{1}\right)^{T} P_{1} \\
& +C_{1}^{T}\left(I-D_{1} R_{1}^{-1} D_{1}^{T}\right) C_{1} \\
& -P_{1} B_{22} R_{1}^{-1} B_{22}^{T} P_{10} \\
& -P_{10} B_{22} R_{1}^{-1} B_{22}^{T} P_{1} \\
& +P_{10} B_{22} R_{1}^{-1} B_{22}^{T} P_{10}
\end{aligned}
$$

Theorem 3.5: Consider the system $\Sigma$ described by equations (1)-(4). Let $\gamma>0, \alpha>0$ and $\beta>0$ be given constants. Suppose the following

(i) Assumptions A1-A3 and (34) hold;

(ii) The Riccati equation (14) has a stabilizing solution $Y \geq 0$;

(iii) There exist matrices $P_{1}>0, P_{10}>0, P_{2}>0$, $P_{20}>0, Q>0$ and $C_{K}$ such that (28) and the following inequalities hold

$$
\left.\begin{array}{rl}
\Delta_{Y}\left(P_{1}, P_{10}, C_{K}, \gamma\right) & <0 \\
\Delta_{K Y}\left(P_{2}, P_{20}, C_{K}, \beta\right) & <0 \\
-Q & C_{0}+D_{0} C_{K} \\
C_{0}^{T}+C_{K}^{T} D_{0}^{T} & -\frac{1}{\gamma^{2}} P_{1}
\end{array}\right]<0
$$

Then the dynamic output feedback controller $K$ described by (5) and (6) with the $C_{K}$ and (24) solves the suboptimal $\beta$-stable mixed $H_{2} / H_{\infty}$ control problem.
Corollary 3.6: With all assumptions as in Theorem 3.5 , except that inequality (40) is replaced by

$$
\begin{gathered}
\Delta_{K Y r}\left(P_{2}, P_{20}, C_{K}, r\right)=\left[\begin{array}{c}
2 r P_{2}+\Delta_{K Y 11}\left(P_{2}, P_{20}\right) \\
C_{K}+B_{22}^{T} P_{2} \\
C_{K}^{T}+P_{2} B_{22} \\
-I
\end{array}\right]<0(42)
\end{gathered}
$$

where $r \geq 0$ is a constant. Then the controller $K$ given by (5) and (6) with the above $C_{K}$ and (24) is with the prescribed degree of stability $r$, and stabilizes the system $\Sigma$ and $J(\Sigma, K, \gamma)<\alpha$.

Remark 3.7: The sufficient condition of Theorem 3.5 is weaker than that of Theorem 3.2, and Theorem 3.5 contains no conservativeness from the assumption on the existence of a common Lyapunov function for $\Sigma_{Y}$ and $\Sigma_{K Y}$ with $u=C_{K} x$. However, it should be mentioned that Theorem 3.5 is still a sufficient condition for the stable controller design problem. There might be no solution if the order of the controller is restricted to that of the plant. In comparison with the condition given in terms of LMIs in Theorem 3.2, the inequalities (28), and (39)-(41) normally are not LMIs, which cannot be solved directly. This class of matrix inequality conditions was used in [10] for the simultaneous linearquadratic optimal control via static output feedback. But, when $P_{10}$ and $P_{20}$ are given, then (28) and (39)(41) are LMIs with respect to the variables $P_{1}, P_{2}, C_{K}$, $Q$ and $\beta^{2}$. This property can be used to form the following convergent iterative algorithms.

First, by combining Theorem 3.2 and Theorem 3.5, we have the following iterative algorithm to minimize $J(\Sigma, K, \gamma)$ for given $\gamma>0$ and $\beta>0$.

Algorithm 3.8: Let $\gamma>0$ and $\beta>0$ be given constants.

Step 1. Solve the Riccati equation (14) to obtain the stabilizing solution $Y \geq 0$.

Step 2. Minimize Trace $(Q)$ subject to the LMI constraints (26), (27) and (29), and denote $C_{K o p t}=$ $W_{\text {opt }} X_{\text {opt }}^{-1}$.

Step 3. Minimize $\beta_{0}^{2}$ subject to $X_{0}>0$ and the LMI constraint (45) below; and minimize Trace $\left(Q_{0}\right)$ subject to LMI constraints (43) and (44).

$$
\begin{aligned}
& {\left[\begin{array}{cc}
M_{c} & X\left(C_{1}^{T}+C_{K o p t}^{T} D_{1}^{T}\right) \\
\left(C_{1}+D_{1} C_{K o p t}\right) X & -\gamma^{2} I
\end{array}\right]<\alpha(43)} \\
& {\left[\begin{array}{cc}
-Q_{0} & \left(C_{0}+D_{0} C_{K o p t}\right) X \\
X\left(C_{0}+D_{0} C_{K o p t}\right)^{T} & -X
\end{array}\right]<0(44)} \\
& {\left[\begin{array}{cc}
M_{d} & X_{0} C_{K o p t}^{T} \\
C_{K o p t} X_{0} & -\beta_{0}^{2} I
\end{array}\right]<}
\end{aligned}
$$

where $M_{c}=\left(A_{11}+B_{22} C_{K o p t}\right) X+X\left(A_{11}+\right.$ $\left.B_{22} C_{K o p t}\right)^{T}+B_{11} B_{11}^{T}$, and $M_{d}=\left(A_{12}+B_{22} C_{K o p t}\right) X_{0}+$ $X_{0}\left(A_{12}+B_{22} C_{K o p t}\right)^{T}+B_{11} B_{11}^{T}$. Denote $P_{10}^{0}=\gamma^{2} X_{o p t}^{-1}$ and $P_{20}^{0}=\beta_{0 o p t}^{2} X_{0 o p t}^{-1}$. 
Step 4. Minimize Trace $\left(Q^{j}\right)$ subject to $P_{2}^{j}>0$ and the LMI constraints

$$
\begin{aligned}
& \Delta_{Y}\left(P_{1}^{j}, P_{10}^{j}, C_{K}^{j}, \gamma\right)<0, \quad \Delta_{K Y}\left(P_{2}^{j}, P_{20}^{j}, C_{K}^{j}, \beta\right)<0 \\
& {\left[\begin{array}{cc}
-Q^{j} & C_{0}+D_{0} C_{K}^{j} \\
\left(C_{0}+D_{0} C_{K}^{j}\right)^{T} & -\frac{1}{\gamma^{2}} P_{1}^{j}
\end{array}\right]<0}
\end{aligned}
$$

where $P_{10}^{j}=P_{1 \text { opt }}^{j-1}, P_{20}^{j}=P_{2 o p t}^{j-1}, j=1,2, \cdots$, and $P_{1 \text { opt }}^{j-1}$ and $P_{2 o p t}^{j-1}$ are the solutions of the $(j-1)$ th optimization. When Trace $\left(Q_{\text {opt }}^{j-1}\right)-\operatorname{Trace}\left(Q_{\text {opt }}^{j}\right)<\epsilon$ for some $\epsilon>0$, stop.

Remark 3.9: In Algorithm 3.8, Step 2 and Step 3 provide initial solutions $P_{10}^{0}$ and $P_{20}^{0}$ for the iterative computation in Step 4 . It is easy to see that $\operatorname{Trace}\left(Q_{o p t}^{j}\right) \leq \operatorname{Trace}\left(Q_{o p t}^{j-1}\right), j=1,2, \cdots$, so the sequence $\left\{\text { Trace }\left(Q_{o p t}^{j}\right)\right\}_{j=1}^{\infty}$ is convergent, which implies that for any $\epsilon>0$, the inequality $\operatorname{Trace}\left(Q_{o p t}^{j-1}\right)-$ Trace $\left(Q_{\text {opt }}^{j}\right)<\epsilon$ will be satisfied for some large enough $j$. So the algorithm is convergent with respect to the optimization objective Trace $\left(Q^{j}\right)$. The algorithm is based on the combination of Theorem 3.2 and Theorem 3.5; the iterative part of it (Step 4 in Algorithm 3.8) from Theorem 3.5 will improve the trade-off between $\alpha, \gamma$ and $\beta$, which will be illustrated in Section 5 by an example.

Similarly, combining Corollary 3.3 and Corollary 3.6, we have the following convergent algorithm to minimize $J(\Sigma, K, \gamma)$ under the constraints of $\left\|T_{z_{1} w}(K)\right\|_{\infty}<\gamma$ and $K(s)$ with the prescribed degree of stability $r$.

Algorithm 3.10: Let $\gamma>0$ and $r>0$ be given constants.

Step 1. Solve the Riccati equation (14) to obtain the stabilizing solution $Y \geq 0$.

Step 2. Minimize Trace $(Q)$ subject to the LMI constraints (26), (27) and (31). Denote $C_{K o p t}=W_{o p t} X_{o p t}^{-1}$. Step 3. Minimize Trace $\left(Q_{0}\right)$ subject to the LMI constraints (43), (44); and minimize Trace $\left(P_{2}\right)$ subject to $P_{2}>0$ and the LMI constraint

$$
\begin{array}{r}
P_{2}\left(A_{12}+r I+B_{22} C_{K o p t}\right)+( \\
\left.A_{12}+r I+B_{22} C_{K o p t}\right)^{T} P_{2} \\
+C_{K o p t}^{T} C_{K o p t}<0 \quad(46)
\end{array}
$$

Denote $P_{10}^{0}=\gamma^{2} X_{o p t}^{-1}$ and $P_{20}^{0}=P_{2 o p t}$.

Step 4. Minimize Trace $\left(Q^{j}\right)$ subject to $P_{2}^{j}>0$ and the LMI constraints

$$
\begin{aligned}
& \Delta_{Y}\left(P_{1}^{j}, P_{10}^{j}, C_{K}^{j}, \gamma\right)<0, \quad \Delta_{K Y \tau}\left(P_{2}^{j}, P_{20}^{j}, C_{K}^{j}, r\right)<0 \\
& {\left[\begin{array}{cc}
-Q^{j} & C_{0}+D_{0} C_{K}^{j} \\
\left(C_{0}+D_{0} C_{K}^{j}\right)^{T} & -\frac{1}{\gamma^{2}} P_{1}^{j}
\end{array}\right]<0}
\end{aligned}
$$

where $P_{10}^{j}=P_{1 o p t}^{j-1}, P_{20}^{j}=P_{2 o p t}^{j-1}, j=1,2, \cdots$, and $P_{1 \text { opt }}^{j-1}$ and $P_{2 \text { opt }}^{j-1}$ are the solutions of the $(j-1)$ th optimization. When Trace $\left(Q_{o p t}^{j-1}\right)-\operatorname{Trace}\left(Q_{o p t}^{j}\right)<\epsilon$ for some $\epsilon>0$, stop.
Remark 3.11: In [8], sufficient conditions for the stable mixed $H_{2} / H_{\infty}$ control problem are given in terms of solutions to three coupled Riccati equations, which are difficult to solve. Comparing with the results in [8], Theorem 3.2 and Theorem 3.5 are given in terms of a Riccati equation and matrix inequalities by solving a multiobjective control problem, and convergent iterative algorithms are developed based on the two theorems. Moreover, the performances such as $H_{\infty}$ norm and prescribed degree of stability of the designed controller are addressed, which are not covered in [8].

\section{Example}

In this section, we will present an example to illustrate the proposed algorithms.

Example 5.1: The example is to illustrate the use of Algorithm 3.10 to design a mixed $H_{2} / H_{\infty}$ controller with a prescribed degree of stability $r$. The system model is as follows.

$$
\begin{gathered}
A=\left[\begin{array}{cc}
-1 & 0.5 \\
0.5 & -1
\end{array}\right], \quad B_{2}=\left[\begin{array}{l}
0 \\
1
\end{array}\right], \quad C_{2}=\left[\begin{array}{ll}
1 & 0
\end{array}\right] \\
B_{1}=\left[\begin{array}{ll}
0 & 0 \\
1 & 0
\end{array}\right], \quad C_{1}=\left[\begin{array}{ll}
1 & 0 \\
0 & 0
\end{array}\right], \quad D_{1}=\left[\begin{array}{l}
0 \\
1
\end{array}\right] \\
D_{2}=\left[\begin{array}{ll}
0 & 1
\end{array}\right], \quad C_{0}=C_{1}, \quad D_{0}=\left[\begin{array}{l}
0 \\
1
\end{array}\right]
\end{gathered}
$$

Let $\gamma=0.5$, by using the result in [9] and LMI Control Toolbox [4], the optimal mixed $H_{2} / H_{\infty}$ controller as shown in Table 1 . The mixed $H_{2} / H_{\infty}$ cost $J_{\text {opt }}$ (= $J_{d}=J_{a}$ ) of the closed-loop system is 0.0842 , and the stability degree of the controller is less than 0.4816 .

Consider the problem of designing an $\mathrm{H}_{2} / \mathrm{H}_{\infty}$ controller with the prescribed degree $r$ of stability for $r=1$. By using Algorithm 3.10 without Step 4 and with Step 4 (200 iterations), the computed results are shown in Table 1.

Table 1: Comparative Results for Example 1 with $\gamma=0.5$

\begin{tabular}{|l|c|}
\hline \hline & Controller Parameters \\
\hline Optimal $H_{2} / H_{\infty}$ & $A_{K m}, B_{K m}, C_{K m}, D_{K m}$ \\
\hline Algorithm 3.10N & $A_{K m 1}, B_{K m 1}, C_{K m 1}, D_{K m 1}$ \\
\hline Algorithm 3.10 & $A_{K m 2}, B_{K m 2}, C_{K m 2}, D_{K m 2}$ \\
\hline
\end{tabular}

\begin{tabular}{|l|c|c|}
\hline \hline$J_{d}$ & $J_{a}$ & Poles of Controller \\
\hline 0.0842 & 0.0842 & $-0.4816,-1.5490$ \\
\hline 0.1068 & 0.1004 & $-1.2820 \pm 0.3694 i$ \\
\hline 0.0888 & 0.0888 & $-1.005,-1.1393$ \\
\hline
\end{tabular}

where Algorithm $3.10 \mathrm{~N}$ represents Algorithm 3.10 without Step $4 ; J_{d}$ denotes the designed mixed $H_{2} / H_{\infty}$ 
cost; and $J_{a}$ denotes the actually achieved mixed $H_{2} / H_{\infty}$ cost.

From Table 1, the results obtained via Algorithm 3.10 without Step 4 are conservative, where $J_{a} \neq J_{d}$ and the controller has much larger stability degree than the designed value 1. However, Algorithm 3.10 with Step 4 gives better results, where $J_{a}=J_{d}$ and the controller has only a slightly larger stability degree than the designed value 1 . Comparing with the optimal mixed $\mathrm{H}_{2} / \mathrm{H}_{\infty}$ cost $J_{\text {opt }}$, it is easy to see that the expense of increasing the stability degree of the controller (from -0.4816 to -1.005 ) is an increase of $5.34 \%$ (from 0.0842 to 0.0887 ) in the optimal mixed $H_{2} / H_{\infty}$ cost. The controller parameters are given in the Appendix.

Acknowledgment- This work is supported by the Academic Research Fund of the Ministry of Education, Singapore, under grant MID-ARC 3/97.

\section{References}

[1] Boyd, S., L. El Ghaoui, E. Feron and V. Balakrishan, Linear Matrix Inequalities in Systems and Control Theory, Philadelphia, PA: SIAM, 1994.

[2] Cao, -Y.Y. and J. Lam, "On simultaneous $H_{\infty}$ control and strong $H_{\infty}$ stabilization," Automatica, Vol. 36, No. 6, (to appear), 1999.

[3] Doyle, J.C., K. Glover, P.P. Khargonekar, and B.A. Francis, "State space solutions to standard $\mathrm{H}_{2}$ and $H_{\infty}$ control problems," IEEE Transactions on $\mathrm{Au}$ tomatic Control, Vol. AC-34, pp. 831-847, 1989.

[4] Gahinet, P., A. Nemirovski, A.J. Laub, and M. Chilali, LMI Control Toolbox, Natick, MA: The MathWorks, 1995.

[5] Ganesh, C. and J.B. Pearson, " $H_{2}$-optimization with stable controllers," Automatica, Vol. 25, pp. 629 634, 1989.

[6] Ito, H., H. Ohmori, and A. Sano, "Design of stable controllers attaining low $H_{\infty}$ weighted sensitivity," IEEE Transactions on Automatic Control, Vol. AC-38, pp. $485-488,1993$.

[7] Jacobus, M., M. Jamshidi, C. Abdallah, P. Dorato, and D. Bernstein, "Suboptimal strong stabilization using fixed-order dynamic compensation," Proc. 1990 Amer. Contr. Conf., San Diego, CA, pp. 2659$2660,1990$.

[8] Kapila, V. and V.M. Haddad, " $\mathrm{H}_{2}$ and mixed $\mathrm{H}_{2} / \mathrm{H}_{\infty}$ stable stabilization," Proc. IEEE Conference on Decision and Control, New Orleans, LA, pp. 19111916, 1995.

[9] Khargonekar, P.P. and M.A. Rotea, "Mixed $H_{2} / H_{\infty}$ control: a convex optimization approach," IEEE Transactions on Automatic Control, Vol. AC-36, no.7, pp. 824-837, 1991.
[10] Lam, J. and Y.Y. Cao, "Simultaneous linearquadratic optimal control design via static output feedback", Int. J. Robust Nonlinear Control, Vol. 9, pp. 551-558, 1999.

[11] Nakayama, T., H. Ohmori, A. Sano, and H. Ito, "A design of $H_{\infty}$ stable controller," Proc. European Control Conference, Rome, Italy, pp. 1830-1833, 1995.

[12] Vidyasagar, M., Control System Synthesis: A Factorization Approach, Cambridge, MA, MIT Press, 1995.

[13] Wang, Y.W. and D.S. Bernstein, " $H_{2}$ suboptimal stable stabilization," Proc. IEEE Conference on Decision and Control, San Antonio, TX, pp. 1828-1829, 1993.

[14] Wang, Y.W., W.M. Haddad, and D.S. Bernstein, "Robust strong stabilization via modified Popov controller synthesis," IEEE Transactions on Automatic Control, Vol. AC-39, no.11, pp. 2284-2287, 1994.

[15] Yang, G.H., J.L. Wang, Y.C. Soh and J. Lam, "Stable controller synthesis for linear time-invariant systems", submitted to International Journal of Control, 2001.

[16] Youla, D.C., J.J. Bougiorno, and C.N. Lu, "Single-loop feedback stabilization of linear multivariable dynamical plants," Automatica, Vol. 19, pp. 159$173,1974$.

[17] Zeren, M. and H. Ozbay, "On the strong stabilization problem and performance of stable $H_{\infty}$ controllers," Proc. 36th IEEE Conference on Decision and Control, San Diego, CA, pp. 4635-4640, 1997.

[18] Zeren, M. and H. Ozbay, "On the synthesis of stable $H_{\infty}$ controllers," IEEE Transactions on Automatic Control, Vol. AC-44, no.2, pp. 431-435, 1999.

\section{Appendix: Controller Parameters}

Controller parameters for Example 5.1:

$$
\begin{gathered}
A_{K m}=\left[\begin{array}{cc}
-0.8077 & 0.5000 \\
0.4835 & -1.2229
\end{array}\right], \quad B_{K m}=\left[\begin{array}{l}
0.0641 \\
0.1540
\end{array}\right] \\
C_{K m}=\left[\begin{array}{ll}
-0.4785 & -0.2229
\end{array}\right] \\
D_{K m}=0 \\
A_{K m 1}=\left[\begin{array}{cc}
-0.8077 & 0.5000 \\
-0.7228 & -1.7563
\end{array}\right], \quad B_{K m 1}=\left[\begin{array}{l}
0.0641 \\
0.1540
\end{array}\right] \\
C_{K m 1}=\left[\begin{array}{ll}
-1.6847 & -0.7563
\end{array}\right] \\
D_{K m 1}=0 \\
A_{K m 2}=\left[\begin{array}{cc}
-0.8077 & 0.5000 \\
-0.1313 & -1.3372
\end{array}\right], \quad B_{K m 2}=\left[\begin{array}{l}
0.0641 \\
0.1540
\end{array}\right] \\
C_{K m 2}=\left[\begin{array}{ll}
-1.0932 & -0.3372
\end{array}\right] \\
D_{K m 2}=0
\end{gathered}
$$

